\title{
Design Study of a Thermocouple Power Sensor as a Monolithic Fin-line
}

\author{
Mark H. Jones, Student Member, IEEE, and Prof. Jonathan Scott, Senior Member, IEEE
}

\begin{abstract}
Making traceable power measurements above $110 \mathrm{GHz}$ using current measurement technologies is challenging. We investigate a design of power sensor consisting of a thermocouple-based integrated circuit (IC) mounted as a finline component in WR-6 waveguide. The design is original in that it contains an antenna, terminating resistor and thermocouples on-chip. We detail the design and report results from simulations and measurements made on a two-port 16:1 scale model. Our design of scale model provides both insertion and reflection loss measurements. Electromagnetic simulation and easily-calibrated model measurements confirm that the short antenna fins feasible on a monolithic microwave integrated circuit (MMIC) can achieve acceptable specifications. The design proves to be relatively insensitive to the value of the terminating resistance or the size of the antenna fins.
\end{abstract}

\section{INTRODUCTION}

The current range of electromagnetic (EM) power sensors employ one, or a combination, of either diode, thermistor or thermocouple type sensing elements [1]. Electromagnetic power measurement took a major step forward in 1974 when an EM power sensor was made using monolithic metal-silicon thermocouples [2]. Integrated thermocouple technology was eventually adopted by all major manufacturers as a result of the design [3]-[5]. The thermocouple sensing elements proposed in this study use fabrication technologies developed for Jackson's design [2]. Monolithic metal-semiconductor thermocouples have been optimised for use on GaAs substrates [6] and offer a robust approach for thermal sensing, desirable when moving into unknown territory.

The standard waveguide for the frequency band $110-170 \mathrm{GHz}$ is WR-6. WR-6 waveguide has internal dimensions small enough to allow a microchip to be fitted directly within the guide cavity. Work has been done toward packaging ICs as finline components directly in waveguide but the idea is not commonplace [7]. The use of an IC as an integrated finline component is a new approach made possible by the small dimensions of waveguide at this frequency range.

\section{SENSOR DESIGN}

The idea is to mount an IC as a finline component in a one port waveguide bulkhead. To permit the measurement of power, the IC would contain an antenna, thin-film terminating resistor and an array of thermocouples. Electromagnetic energy that enters the bulkhead would be absorbed by the antenna and dissipated as heat in the terminating resistor, which would be sensed by the array of thermocouples. The waveguide would be made in 'clamshell-like' halves, between which the IC would be placed during assembly.
(A) Thin-film resistor (B) Antenna (C) Thermocouple hot-junction

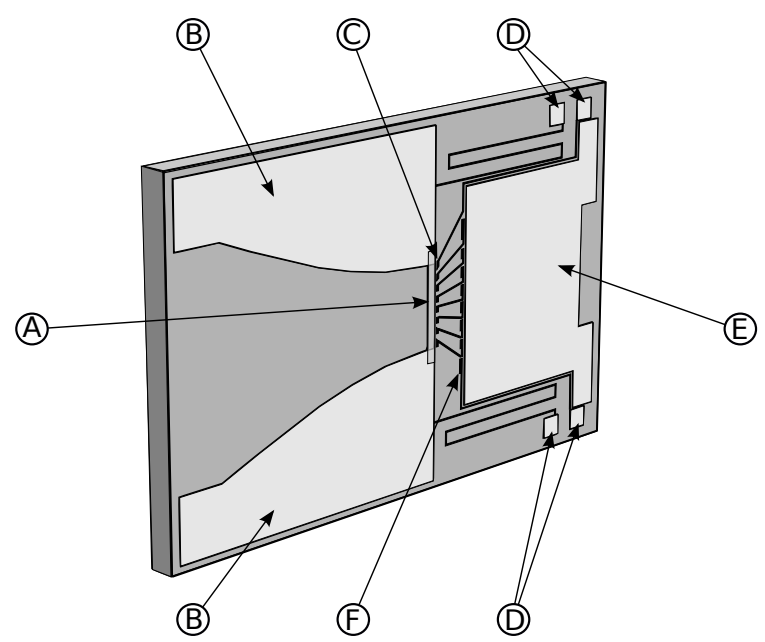

(D) Pads (E) Heat Spreader (F) Thermocouple cold-junction

Fig. 1. Diagram of the microchip intended for use in millimetre wave power measurement

WR-6 waveguide has a cross section of $1650 \mu \mathrm{m}$ by $825 \mu \mathrm{m}$ $(0.065$ " by 0.0325 "). Therefore, an IC to be mounted in WR-6 waveguide must have one dimension slightly more than $825 \mu \mathrm{m}$. It is practical to make an IC only a few times longer than it is wide (to prevent breakages). In this investigation we envisage an IC that is approximately $1300 \mu \mathrm{m}$ high and $2100 \mu \mathrm{m}$ long ( $0.0512 "$ by 0.0823 "), with the central strip of $825 \mu \mathrm{m}$ being the 'active' area that is exposed to the waveguide cavity. The side wings will provide a mounting area as well as a thermal escape route for dissipated heat. A diagram of such a circuit appears in figure 1. In this diagram, thermocouples are placed between a heat spreader at the rear of the chip and the terminating resistance between the antenna fins. Etching away epitaxial layers from the underside of the chip, as is seen in Jackson's design [2], will increase thermal isolation of the resistor and hot thermocouple junctions, leading to an increase in sensitivity.

\section{Measurement Details And Results}

Although the final design of the sensor would be a oneport bulkhead, a two-port version was studied. A two-port model allows measurement of both energy reflected from and transmitted past the finline mounted IC. A 16:1 scale model of the IC and waveguide section was fabricated and is depicted 
(A) Coax-Waveguide Transition

(B) Clamshell centre section

(C) PCB Layout of microchip

(D) Female N-type connector

(E) $60 \mathrm{~mm}$ M6 Bolts

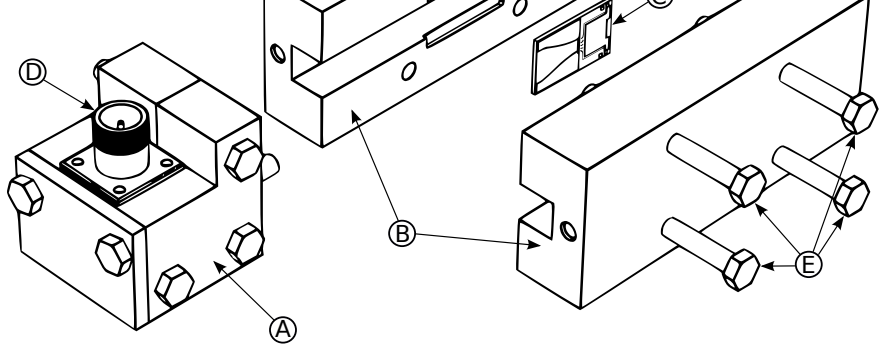

(A)

Fig. 2. Exploded diagram of scale model built for simulation verification.

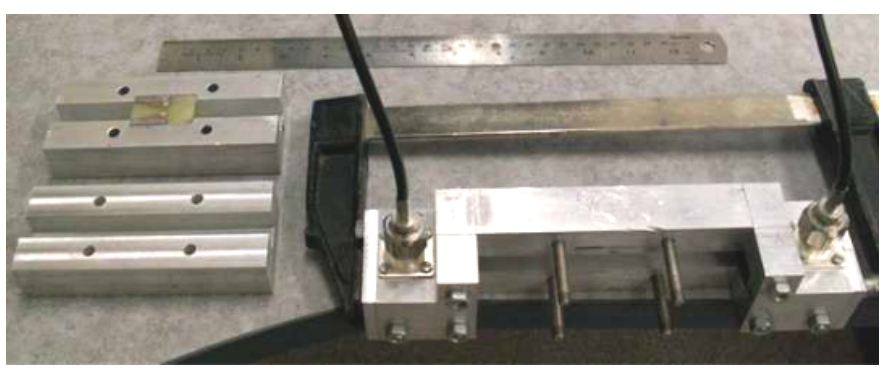

Fig. 3. Picture of the scale model with interchangeable clamshell parts for calibration visible alongside.

in figures 2 and 3 . The $16: 1$ scale for the measurement model was chosen as this matches the relative thicknesses of the semiconductor substrate and metallisation layer $(100 \mu \mathrm{m}$ and $2 \mu \mathrm{m}$ respectively) to the thicknesses of a standard 1oz. printed circuit board (PCB) fibreglass and copper layers $(1.6 \mathrm{~mm}$ and $35.6 \mu \mathrm{m}$ respectively). Using a large scale model allows for more relaxed fabrication tolerances while still maintaining relative tolerances between the IC and waveguide section.

The model was built in three sections, each of which were formed from aluminium halves. The interior dimensions of the model waveguide cavity were $26.42 \mathrm{~mm}$ by $13.21 \mathrm{~mm}$ (1.040" by 0.520 "). This resulted in the operational frequency range of $110-170 \mathrm{GHz}$ (WR-6) being scaled down to $6.88-10.63 \mathrm{GHz}$. Measurements of the scale model were made using an Agilent E8358 PNA. The E8358 measures to a maximum of $9 \mathrm{GHz}$, limiting model measurement data to the lower $57 \%$ of the scaled operational frequency range. Surface mount resistors were soldered upside down between the fins to replicate the thin-film terminating resistor of the IC.

Calibrating the E8358 to use the models coaxial to waveguide transitions and waveguide section required reassembling the setup in various configurations in order to satisfy calibration parameter assumptions. The calibration method used was Thru, Reflect, Line (TRL) for which the models centre section was used as the line component. Using the models centre section during calibration was preferable as this meant that the model did not have to be completely disassembled after calibration and made use of already fabricated sections. However, doing so has led to periodic areas of uncertainty within the measurement data. This is due to the line section being longer than a quarter wavelength, which the calibration assumed was being used. This means that multiple solutions are possible at certain points in the calibration procedure, leading to spurious corrections being applied to measurement data at these points. These areas where the measurements have been disturbed by the calibration have been marked with vertical grey bands on the measurement graphs for clarity.

We have assumed that energy that entered the waveguide but did not exit either of the two ports was dissipated as heat within the terminating resistor. This assumption allows the amount of power dissipated as heat in the resistor to be determined by loss factor calculations. The loss factor $(\eta)$ represents the fraction of power that entered the waveguide section and did not leave either of the two ports. The forward loss factor $(\eta)$ is calculated from S-parameters $\left(S_{11}\right.$ and $\left.S_{21}\right)$ as follows:

$$
\eta=1-\left|S_{11}\right|^{2}-\left|S_{21}\right|^{2}
$$

Model simulation was carried out using COMSOL Multiphysics V3.2. The simulations consisted of a centre section, as was used in the scale model, in which the IC was mounted as a finline component. This simulation model was of 1:1 scale and was simulated across the target frequency range (110$170 \mathrm{GHz}$ ). Simulations involve breaking the model up into a mesh of tetrahedrons, each having electromagnetic fields computed over discrete time intervals in order to simulate wave propagation. The meshing becomes an issue for objects that are thin and long such as the fins of the IC, which are over 500 times longer than they are thick and 826,000 times thinner than the width of the waveguide cavity. The large range of dimensions within the model meant that it could not be simulated on available hardware without simplification.

We made the simplification that the fins of the IC and the walls of the waveguide cavity were perfectly conducting. This allowed us to remove all mesh elements from within the fins and outside of the waveguide walls. Model complexity was further reduced by increasing the thickness of the fins from $2 \mu \mathrm{m}$ to $10 \mu \mathrm{m}$, allowing a computable solution.

The simplifications made leave a gap in our understanding of the sensor's performance. Any loss of power attributed to the fins themselves is not understood, which will be an important aspect of the sensor's overall performance. As we, the authors, were mostly interested in the effects of certain design parameters of the IC, namely the terminating resistance value, the affect of this was not investigated.

Figure 4 shows the results for return loss measurements. The three resistance values give similar results indicating that their relative values contribute little when compared to the inclusion of the substrate. Simulated and measured data agree in light of the simplifications made.

Figure 5 clearly shows the effect of the fins and terminating resistance and that the substrate alone is responsible for very little power dissipation. Again the low sensitivity to the value of the terminating resistor value is evident.

Figure 6 shows that one half to three quarters of incident 


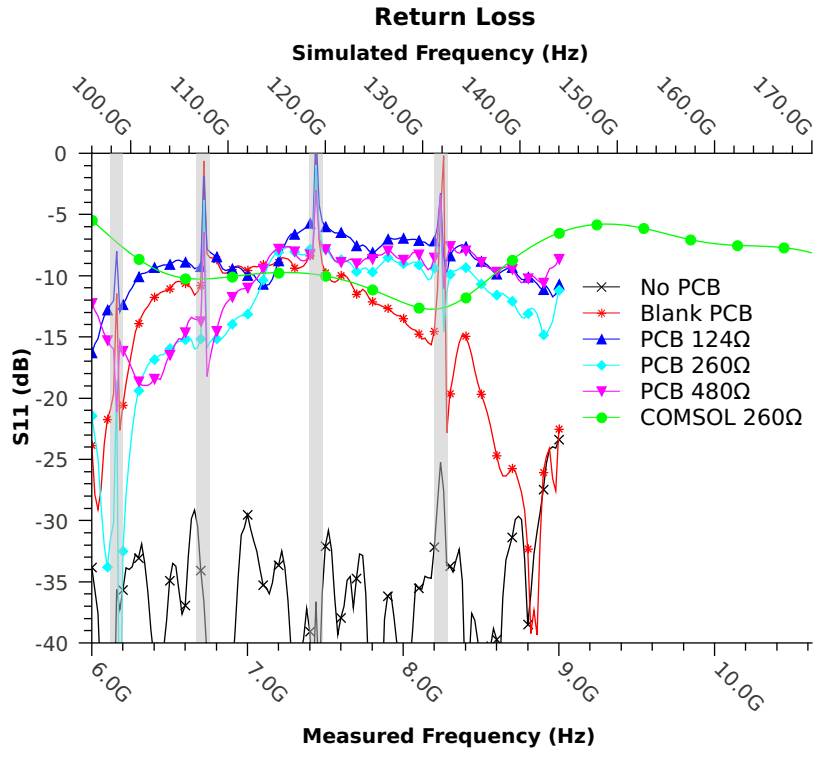

Fig. 4. Simulated and measured insertion loss of the models. The vertical grey bars indicate areas where the calibration fails to provide usable measurements. 'No PCB' refers to an empty waveguide section, 'Blank PCB' refers to a PCB with no fins or resistor and the others refer to an IC with fins and resistances as indicated.

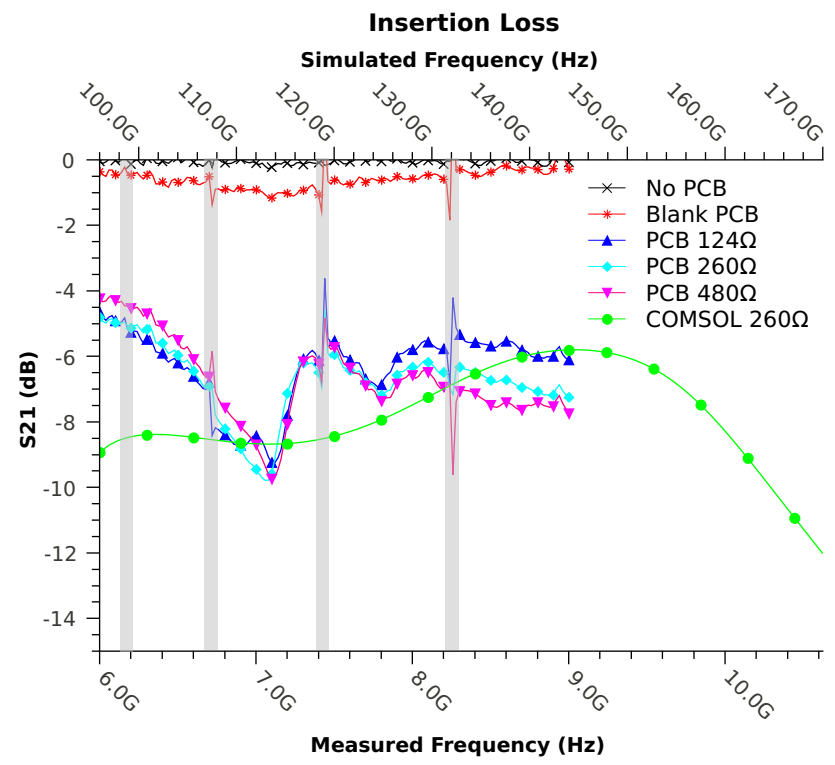

Fig. 5. Simulated and measured return loss of the models. The vertical grey bars indicate areas where the calibration fails to provide usable measurements. 'No PCB' refers to an empty waveguide section, 'Blank PCB' refers to a PCB with no fins or resistor and the others refer to an IC with fins and resistances as indicated.

power is dissipated by the model. An optimum value of terminating resistor lies around $260 \Omega$, with subsequent simulations predicting an optimum slightly below $260 \Omega$. There is considerable agreement as to the magnitude of power loss between simulated and measured data.

\section{CONCLUSION}

It is predicted that a thermally based finline MMIC power sensor should be capable of dissipating at least $50 \%$ or $-3 \mathrm{~dB}$

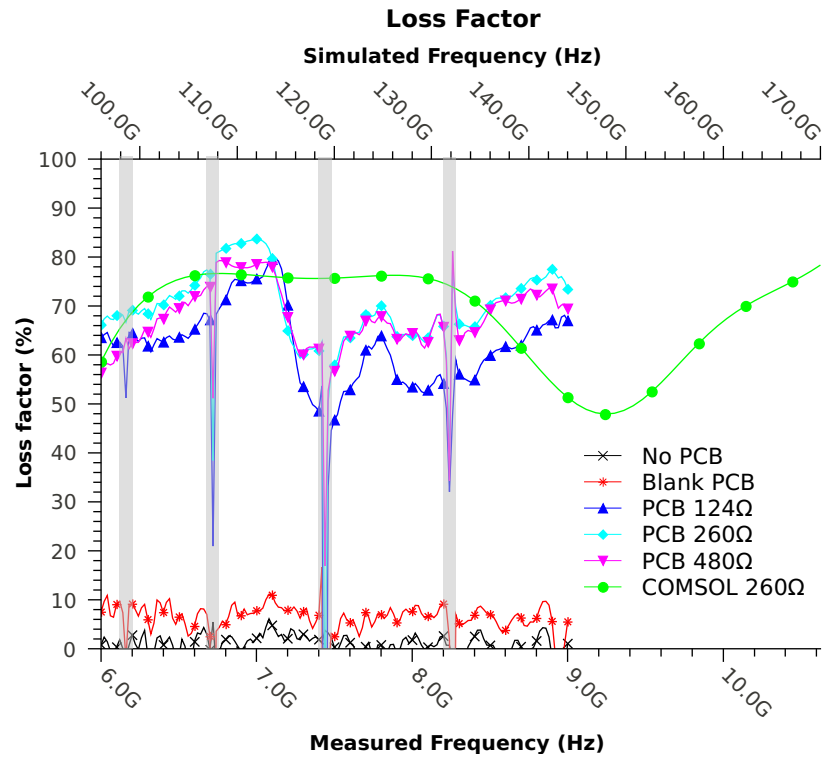

Fig. 6. Simulated and measured loss factor of the models. The vertical grey bars indicate areas where the calibration fails to provide usable measurements. 'No PCB' refers to an empty waveguide section, 'Blank PCB' refers to a PCB with no fins or resistor and the others refer to an IC with fins and resistances as indicated.

of incident power as heat on-chip. Simulation of the sensor is difficult due to the relative mechanical dimensions but simplifications allow a computable solution. Measurements of a scale model indicate that the substrate alone is responsible for considerable relection of energy. A separate investigation shows that optimising the shape and size of the fins, as well as offsetting the chip in the waveguide cavity gives little improvement [8].

\section{ACKNOWLEDGEMENTS}

The authors wish to acknowledge the generous support of Agilent Technologies through a University Research Grant. Thanks also go to Eric Breakenridge of Agilent's South Queensferry site and staff at the Santa Rosa Technology Center. Suren Singh of Agilent's Component Test Division donated equipment for which the authors are grateful.

\section{REFERENCES}

[1] Agilent Technologies, Appl. Note 64-1C, pp.18-47.

[2] W. H. Jackson. "A thin-film semiconductor therocouple for microwave power measurements." HP Journal, vol. 26, September 1974.

[3] H. Delfs and T Betz, "Power measuring sensor for measuring highfrequency power," European Patent: 0223163/B1, June. 1988.

[4] S. Kodato, T. Wakabayashi, Q. Zhuang, and S. Uchida, "New structure for DC-60 GHz thermal power sensor," IEEE MTT International Microwave Symposium Digest, pp. 781-874, June 1996.

[5] S. Kodato, "Wide-band RF signal power detecting element and power detecting device using the same," U.S. Patent 6,518,743 B1, Feb. 2003.

[6] J. B. Scott, T. S. Low, S. Cochran, B. Keppeler, J. Staroba and B. Yates, "New thermocouple-based microwave/mmWave power sensor MMIC techniques in GaAs," IEEE Transactions on Microwave Theory and Techniques, to be published.

[7] A. K. Jastrzebski, "A simple condition for maximum bandwidth of a chip packaged in an mm-wave waveguide," presented at 32nd European Microwave Conference, Milan, Italy, 2002.

[8] M. H. Jones, "Millimeter wave power measurement," M.E. thesis, University of Waikato, New Zealand, 2010. 